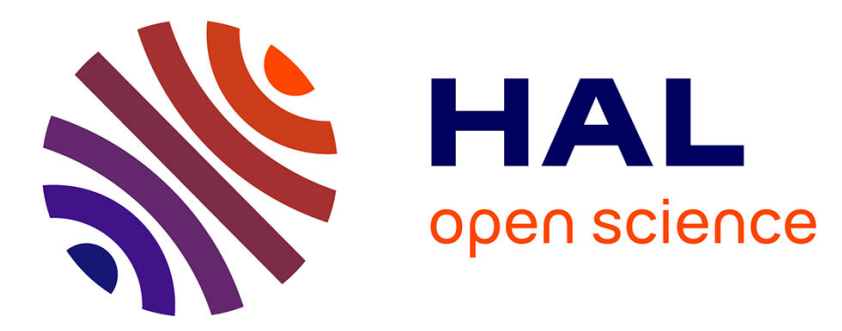

\title{
Polyhedral Regions of Stability for Aperiodic Sampled-data Linear Control Systems with Saturating Inputs
}

\author{
Daniel Denardi Huff, Mirko Fiacchini, Joao Manoel Gomes da Silva
}

\section{- To cite this version:}

Daniel Denardi Huff, Mirko Fiacchini, Joao Manoel Gomes da Silva. Polyhedral Regions of Stability for Aperiodic Sampled-data Linear Control Systems with Saturating Inputs. IEEE Control Systems Letters, 2021, 6, pp.241-246. 10.1109/LCSYS.2021.3066132 . hal-03188711

\section{HAL Id: hal-03188711 \\ https://hal.univ-grenoble-alpes.fr/hal-03188711}

Submitted on 1 Jul 2021

HAL is a multi-disciplinary open access archive for the deposit and dissemination of scientific research documents, whether they are published or not. The documents may come from teaching and research institutions in France or abroad, or from public or private research centers.
L'archive ouverte pluridisciplinaire HAL, est destinée au dépôt et à la diffusion de documents scientifiques de niveau recherche, publiés ou non, émanant des établissements d'enseignement et de recherche français ou étrangers, des laboratoires publics ou privés. 


\title{
Polyhedral Regions of Stability for Aperiodic Sampled-data Linear Control Systems with Saturating Inputs
}

\author{
Daniel Denardi Huff, Mirko Fiacchini, and João Manoel Gomes da Silva Jr.
}

\begin{abstract}
This work proposes a method to asses the local asymptotic stability and to provide polyhedral estimates of the region of attraction of the origin (RAO) of linear systems under aperiodic sampled-data control and saturating inputs. The approach is based on a discrete-time model that describes the behavior of the system state between consecutive sampling instants. It corresponds to a difference inclusion defined from a partition of the intersampling interval and from the saturated and nonsaturated (SNS) embedding of saturation functions. A method to construct a contractive polyhedral set for this model is proposed. It is shown that this set induces a local Lyapunov function strictly decreasing at the sampling instants and that it is an estimate of the RAO of the continuous-time closed-loop system.
\end{abstract}

Index Terms-Sampled-data control, input saturation, polyhedral sets

\section{INTRODUCTION}

$\mathbf{M}$ OTIVATED by the growing use of embedded controllers in different applications, where a communication protocol is responsible for the transmission of data between computer algorithms, actuators and sensors, the analysis and control design for networked control systems have been addressed in many recent works. In this context, aperiodic sampling can be seen as a modeling abstraction employed to represent, in a theoretical framework, the effect of imperfections on the communication channel such as sampling jitters, fluctuations and, in some cases, packet dropouts [1]. The survey [2] presents several existing methods to analyze the stability of aperiodic sampled-data systems when the dynamics is assumed to be linear. Many among these methods are based on an uncertain discrete-time model that describes the behavior of the system state between consecutive sampling instants (e.g. [3]). The exponential dependence of the transition matrix of this difference inclusion model can be dealt with in different ways, using, for instance, polytopic embeddings [4], [5] or norm bounded uncertainties [6], [7]. In [8] it is shown that the exponential stability of the discrete-time model is equivalent

This study was financed in part by the Coordenação de Aperfeiçoamento de Pessoal de Nível Superior - Brazil (CAPES) Finance Code 001 and by the French National Research Agency in the framework of the "Investissements d'avenir" program (ANR-15-IDEX02). J.M. Gomes da Silva Jr. is also supported by CNPQ, Brazil (grant $P Q-307449 / 2019-0)$.

D.D. Huff and M. Fiacchini are with Gipsa-lab, Université Grenoble Alpes (UGA), Grenoble, France. \{daniel.denardi-huff, mirko.fiacchini\}@gipsa-lab.fr

D.D. Huff and J.M. Gomes da Silva Jr. are with the Grad. Program in Electrical Eng. (PPGEE) and the Dept. of Automation and Energy Systems (DELAE), Universidade Federal do Rio Grande do Sul (UFRGS), Porto Alegre, Brazil. \{daniel. huff, jmgomes\}eufrgs.br to the existence of a polyhedral Lyapunov function for the system.

Moreover, due to physical limitations of actuators, the input saturation is ubiquitous in real control problems. It is a source of performance degradation and, in many cases, only local (or regional) stability of the closed-loop system can be ensured, even for linear plants. In the periodic sampled-data case, as the saturation nonlinearity affects only the input, the stability analysis can be carried out by using a discrete-time model (see [9] and references therein) obtained by exact discretization. Nevertheless, for the aperiodic sampled-data case, the problem is more involved and requires a careful analysis of the hybrid behavior of the system. Regarding this case we can cite [10] and [11], which provide LMI-based techniques to compute ellipsoidal estimates of the region of attraction of the origin (RAO) of the closed-loop system, which can be seen as safe regions of operation.

The present work addresses the same problem of [10], [11], but focussing on the determination of polyhedral estimates of the RAO. The proposed approach, inspired by [12] and [8], relies on the use of a discrete-time model that describes the behavior of the system state between consecutive sampling instants. It is proved that if the discrete-time trajectories converge to the origin then the continuous-time ones also do. This discrete-time model corresponds to a difference inclusion obtained from the use of the saturated and nonsaturated (SNS) embedding for the saturation term [13]. It is shown that contractive sets for this difference inclusion remain contractive when scaled down and can therefore be associated to a Lyapunov function which is strictly decreasing at the sampling instants. It is also shown that the obtained sets can be used as estimates of the RAO of the continuous-time closedloop system. In order to obtain these estimates, a numerical algorithm is proposed that converges to a contractive set for the considered difference inclusion under mild assumptions. As shown by numerical examples, the method provides a powerful tool to compute polyhedral estimates of the RAO of the system under study.

Notation. $\mathbb{N}_{m} \triangleq\{i \in \mathbb{N}: 1 \leq i \leq m\}$ and $\mathscr{S} \triangleq 2^{\mathbb{N}_{m}}$ is the set of all subsets of $\mathbb{N}_{m}$. For instance: $2^{\mathbb{N}_{2}}=\{\emptyset,\{1\},\{2\},\{1,2\}\}$. A C-set $\Omega$ is a compact and convex set containing the origin in its interior. Given a matrix $M, M_{(i)}$ is its $i$-th row, $M^{(i)}$ is its $i$-th column, $M^{T}$ is its transpose and $\|M\|$ is its induced 2-norm. If $M$ is symmetric then $\lambda_{\max }(M)$ is its maximum eigenvalue. The components of $\operatorname{sat}(v): \mathbb{R}^{m} \rightarrow \mathbb{R}^{m}$ are defined as $\operatorname{sat}_{(r)}(v) \triangleq \operatorname{sign}\left(v_{(r)}\right) \min \left\{\left|v_{(r)}\right|, 1\right\}, r \in \mathbb{N}_{m} . \mathscr{P}(H, h) \triangleq$ $\left\{x \in \mathbb{R}^{n}: H x \leq h\right\}, H \in \mathbb{R}^{n_{h} \times n}, h \in \mathbb{R}^{n_{h}}$, corresponds to the $H$ representation of a polyhedron. Given $\Omega \subseteq \mathbb{R}^{n}, \operatorname{Co}(\Omega)$ is its 
convex hull and $\Omega^{\circ}$ its interior. $e_{k}$ is the $k$-th canonical base vector of the Euclidean space and $\mathbf{1} \triangleq[1 \ldots 1]^{T}$.

\section{Problem Formulation}

The system under study is given by the dynamics

$$
\dot{x}(t)=A_{p} x(t)+B_{p} u(t)
$$

where $x \in \mathbb{R}^{n}$ and $u \in \mathbb{R}^{m}$ represent the state and the input of the plant, respectively. Matrices $A_{p}$ and $B_{p}$ have appropriate dimensions and are supposed to be constant. The control input is computed based on the sampled-value of the state at the time instants $t_{k}$, with $k \in \mathbb{N}$, according to

$$
u(t)=\operatorname{sat}\left(K x\left(t_{k}\right)\right), \quad \forall t \in\left[t_{k}, t_{k+1}\right) .
$$

The difference between two successive sampling instants, given by $\delta_{k} \triangleq t_{k+1}-t_{k}$, is considered to be lower and upper bounded as follows:

$$
0<\tau_{m} \leq \delta_{k} \leq \tau_{M}, \forall k \in \mathbb{N} .
$$

Since $\delta_{k}$ depends on $k$, this system models an aperiodic sampling strategy. The particular case of periodic sampling corresponds to $\delta_{k}=\tau_{m}=\tau_{M}$ for all $k \in \mathbb{N}$.

Denoting $x_{k} \triangleq x\left(t_{k}\right)$, it follows, from the analytical solution of (1) considering (2), that the dynamics between two successive sampling instants can be described by the following difference inclusion:

$$
x_{k+1} \in\left\{A(\delta) x_{k}+B(\delta) \operatorname{sat}\left(K x_{k}\right): \delta \in \Delta\right\}
$$

where $\Delta \triangleq\left[\tau_{m}, \tau_{M}\right], A(\delta) \triangleq e^{A_{p} \delta}$ and $B(\delta) \triangleq \int_{0}^{\delta} e^{A_{p} s} \mathrm{~d} s B_{p}$.

Definition 1: Given that the origin of a continuous(discrete-) time system is asymptotically stable, the region of attraction of the origin (RAO) is the set of all $x \in \mathbb{R}^{n}$ such that for $x(0)=x\left(x_{0}=x\right)$ it follows that $\lim _{t \rightarrow \infty} x(t)=0$ $\left(\lim _{k \rightarrow \infty} x_{k}=0\right)$.

The following bound between two sampling instants holds:

$$
\begin{aligned}
\|x(t)\| & \leq\left\|A\left(t-t_{k}\right)\right\|\left\|x\left(t_{k}\right)\right\|+\left\|B\left(t-t_{k}\right)\right\|\left\|\operatorname{sat}\left(K x\left(t_{k}\right)\right)\right\| \\
& \leq\left(\left\|A\left(t-t_{k}\right)\right\|+\left\|B\left(t-t_{k}\right)\right\|\|K\|\right)\left\|x\left(t_{k}\right)\right\| \\
& \leq \max _{\tau \in\left[0, \tau_{M}\right]}(\|A(\tau)\|+\|B(\tau)\|\|K\|)\left\|x\left(t_{k}\right)\right\| \\
& \triangleq C_{A}\left\|x_{k}\right\|, \quad \forall t \in\left[t_{k}, t_{k+1}\right], \quad \forall k \in \mathbb{N}
\end{aligned}
$$

Hence, the asymptotic stability of the origin of the discretetime model (4) ensures the asymptotic stability of the continuous-time system (1)-(2). Then, to obtain estimates of the RAO of the closed-loop system (1)-(2), it suffices to analyze the behavior of the discrete-time model (4), since $x_{k} \stackrel{k \rightarrow \infty}{\rightarrow} 0$ implies that $x(t) \stackrel{t \rightarrow \infty}{\rightarrow} 0$.

As pointed out in [9, Section 1.7], the direct handling of the saturation nonlinearity in (4), in order to obtain testable numerical conditions to assess the asymptotic stability of the origin and to compute estimates of the RAO, is a quite hard task. To overcome this problem, many representations for the saturation term, such as sector bounded nonlinearities [10] and polytopic embeddings [11], have been successfully considered in the literature. Another representation is the saturated and nonsaturated (SNS) one [13], which is less conservative than classical polytopic embeddings. Moreover, as it will be shown in Section III, it is suitable to compute one-step sets. The basic idea in this case is to estimate the RAO of (4) by using the difference inclusion

$$
\begin{aligned}
x_{k+1} & \in\left\{A(\delta) x_{k}+B(\delta) \operatorname{sat}_{S}\left(K x_{k}\right): \delta \in \Delta, S \in \mathscr{S}\right\} \\
& \triangleq\left\{F_{S N S}\left(x_{k}, \delta, S\right): \delta \in \Delta, S \in \mathscr{S}\right\} \triangleq F_{S N S}\left(x_{k}, \Delta, \mathscr{S}\right)
\end{aligned}
$$

where $\operatorname{sat}_{S}(z) \triangleq \sum_{i \in S^{c}} e_{i} z_{(i)}+\sum_{i \in S} e_{i} \operatorname{sat}_{(i)}(z), S \in \mathscr{S}$ and $S^{c}=$ $\mathbb{N}_{m} \backslash S$ (see Notation). The definition of the SNS system (5) involves an additional parameter $S$, denoting the components of the input that saturate. The difference inclusion (5) takes into account all $2^{m}$ possible combinations of saturated/nonsaturated inputs given by $S \in \mathscr{S}=2^{\mathbb{N}_{m}}$ simultaneously. For example, if $m=2$ then $S \in \mathscr{S}=2^{\mathbb{N}_{2}}=\{\emptyset,\{1\},\{2\},\{1,2\}\}$ and one has

$$
\begin{aligned}
& \operatorname{sat}_{\{\emptyset\}}\left(K x_{k}\right)=\left[\begin{array}{l}
K_{(1)} x_{k} \\
K_{(2)} x_{k}
\end{array}\right], \operatorname{sat}_{\{1\}}\left(K x_{k}\right)=\left[\begin{array}{c}
\operatorname{sat}\left(K_{(1)} x_{k}\right) \\
K_{(2)} x_{k}
\end{array}\right] \\
& \operatorname{sat}_{\{2\}}\left(K x_{k}\right)=\left[\begin{array}{c}
K_{(1)} x_{k} \\
\operatorname{sat}\left(K_{(2)} x_{k}\right)
\end{array}\right], \operatorname{sat}_{\{1,2\}}\left(K x_{k}\right)=\left[\begin{array}{l}
\operatorname{sat}\left(K_{(1)} x_{k}\right) \\
\operatorname{sat}\left(K_{(2)} x_{k}\right)
\end{array}\right] .
\end{aligned}
$$

Notice that (5) embeds (4): any trajectory $\left\{x_{k}\right\}_{k=0}^{\infty}$ satisfying (4) belongs to the family of trajectories that satisfy (5). In other words, an estimate of the RAO of (5) will also be a valid estimate of the RAO of (4) and, consequently, an estimate of the RAO of the closed-loop system (1)-(2). Hence, instead of directly computing an estimate for (4), we will aim at obtaining an estimate for (5) using convex analysis tools [14] that cannot be directly applied to (4). This estimate will correspond to a polyhedral contractive C-set, according to the following definition.

Definition 2: ( $\lambda$-contractive set) Given $0 \leq \lambda<1$, the Cset $\Omega \subset \mathbb{R}^{n}$ is said to be $\lambda$-contractive for a generic difference inclusion $x_{k+1} \in G\left(x_{k}\right)$ if $G\left(x_{k}\right) \subseteq \lambda \Omega$ for all $x_{k} \in \Omega$. If $\lambda=1$, $\Omega$ is a (positively) invariant set.

In particular, notice that a $\lambda$-contractive $\mathrm{C}$-set for (5) is also $\lambda$-contractive for (4) but the opposite is not true in general. Moreover, the following key result holds.

Lemma 1: If a $\mathrm{C}$-set $\Omega$ is $\lambda$-contractive for (5) then $\varepsilon \Omega$ is also $\lambda$-contractive for (5) for all $\varepsilon \in \mathbb{R}$ such that $0<\varepsilon \leq 1$.

Proof: Given $\bar{x} \in \varepsilon \Omega$, we have to prove that $F_{S N S}(\bar{x}, \Delta, \mathscr{S})=F_{S N S}(\varepsilon x, \Delta, \mathscr{S}) \subseteq \lambda \varepsilon \Omega$, where $x \triangleq \bar{x} / \varepsilon \in$ $\Omega$. Since $\operatorname{sat}(\varepsilon y) \in \varepsilon \operatorname{Co}\{y, \operatorname{sat}(y)\}$ for $y \in \mathbb{R}$ and $0 \leq \varepsilon \leq$ 1 , it can be verified that, for $S \in \mathscr{S}, z \triangleq \operatorname{sat}_{S}(\varepsilon y) \in$ $\varepsilon \operatorname{Co}\left\{\operatorname{sat}_{S^{\prime}}(y): S^{\prime} \in \mathscr{S}\right\}$ in the multivariable case, i.e. when $y \in \mathbb{R}^{m}$. To see this notice that

$$
z_{(i)}=\left\{\begin{array}{ll}
\operatorname{sat}_{(i)}(\varepsilon y), & \text { if } i \in S \\
\varepsilon y_{(i)}, & \text { if } i \notin S
\end{array},\right.
$$

then $z_{(i)}$ belongs to the interval $I_{i} \triangleq \varepsilon \operatorname{Co}\left\{y_{(i)}, \operatorname{sat}_{(i)}(y)\right\}$ and, consequently, $z$ belongs to the polytope $I \triangleq I_{1} \times \cdots \times I_{m} \subset$ $\mathbb{R}^{m}$. Notice now that $I=\varepsilon \operatorname{Co}\left\{\operatorname{sat}_{S^{\prime}}(y): S^{\prime} \in \mathscr{S}\right\}$ because the elements of $\left\{\varepsilon \operatorname{sat}_{S^{\prime}}(y): S^{\prime} \in \mathscr{S}\right\}$ are the vertices of $I$.

Using the set inclusion above it follows that for all $\delta \in \Delta$ 
and $S \in \mathscr{S}$

$$
\begin{aligned}
F_{S N S}(\varepsilon x, \delta, S) & =A(\delta) \varepsilon x+B(\delta) \operatorname{sat}_{S}(K \varepsilon x) \\
& \in \varepsilon A(\delta) x+B(\delta) \varepsilon \operatorname{Co}\left\{\operatorname{sat}_{S^{\prime}}(K x): S^{\prime} \in \mathscr{S}\right\} \\
& =\varepsilon \operatorname{Co}\left\{A(\delta) x+B(\delta) \operatorname{sat}_{S^{\prime}}(K x): S^{\prime} \in \mathscr{S}\right\} \\
& \subseteq \varepsilon \operatorname{Co}\left\{F_{S N S}(x, \Delta, \mathscr{S})\right\} \subseteq \varepsilon \operatorname{Co}(\lambda \Omega)=\varepsilon \lambda \Omega
\end{aligned}
$$

where the last set inclusion follows from the fact that $\Omega$ is $\lambda$-contractive for (5) and $x \in \Omega$.

From Lemma 1 , it follows that if $\Omega$ is a $\lambda$-contractive $\mathrm{C}$-set w.r.t. (5) then

$$
x_{k} \in \varepsilon \Omega \Rightarrow x_{k+p} \in \lambda^{p} \varepsilon \Omega, \quad 0 \leq \varepsilon \leq 1,
$$

which implies that $x_{k} \stackrel{k \rightarrow \infty}{\rightarrow} 0$ because $\Omega$ is bounded by definition. Moreover, as (5) encompasses (4), (7) also holds for the trajectories of (4) and it becomes clear that $\Omega$ is an estimate not only of the RAO of (5) but also of the RAO of (4).

There is an alternative statement for Definition 2 based on the Minkowski function $\Psi_{\Omega}: \mathbb{R}^{n} \rightarrow \mathbb{R}$ of the C-set $\Omega$, which is given by $\Psi_{\Omega}(x) \triangleq \min \{\alpha \geq 0: x \in \alpha \Omega\}$ [14]. This function satisfies the following properties.

Lemma 2 ([14]): $\Psi_{\Omega}(\cdot)$ is continuous, positive definite, convex, positively homogeneous of order 1 , sub-additive (i.e. $\left.\Psi_{\Omega}\left(x_{1}+x_{2}\right) \leq \Psi_{\Omega}\left(x_{1}\right)+\Psi_{\Omega}\left(x_{2}\right)\right)$ and lower and upper bounded as follows: $m\|x\| \leq \Psi_{\Omega}(x) \leq M\|x\|, m, M>0$.

The concept can be extended to sets. Given a C-set $\Omega \subset \mathbb{R}^{n}$, the Minkowski function of a compact set $\Theta \subset \mathbb{R}^{n}$ is defined as $\Psi_{\Omega}(\Theta) \triangleq \max _{x \in \Theta} \Psi_{\Omega}(x)$.

Thus, the condition in Definition 2 for a C-set $\Omega$ to be $\lambda$ contractive for $x_{k+1} \in G\left(x_{k}\right)$ is equivalent to:

$$
\begin{aligned}
& \Psi_{\Omega}\left(x_{k+1}\right) \leq \lambda, \forall x_{k+1} \in G\left(x_{k}\right), \forall x_{k} \in \Omega \\
\Leftrightarrow & \Psi_{\Omega}\left(G\left(x_{k}\right)\right) \leq \lambda, \forall x_{k} \in \Omega
\end{aligned}
$$

Notice that the Minkowski function of a $\lambda$-contractive $\mathrm{C}$-set $\Omega$ for (5) is a local Lyapunov function for (5) and consequently for (4), since from (7) it follows that:

$$
\Psi_{\Omega}\left(x_{k}\right) \leq 1 \Rightarrow \Psi_{\Omega}\left(x_{k+1}\right) \leq \lambda \Psi_{\Omega}\left(x_{k}\right) .
$$

\section{StABILITY ANALYSIS}

The proposed stability analysis method can be divided in two steps. The first one, presented in Section III-A, consists in finding a contractive polyhedral C-set $\Omega$ for the dynamics

$$
x_{k+1} \in F_{S N S}\left(x_{k}, \Delta_{J}, \mathscr{S}\right)
$$

where $\Delta_{J} \triangleq\left\{\tau_{m}+(j-1) \tau_{J}: j \in \mathbb{N}_{J}\right\}, \tau_{J} \triangleq \frac{\tau_{M}-\tau_{m}}{J}, J \in \mathbb{N}$. Note that (8) considers only a finite subset $\Delta_{J}$ of the interval $\Delta$ and thus (8) is embedded by (5), i.e. $F_{S N S}\left(x_{k}, \Delta_{J}, \mathscr{S}\right) \subseteq$ $F_{S N S}\left(x_{k}, \Delta, \mathscr{S}\right)$. The second step, presented in Section III-B, verifies if the set $\Omega$ found is also contractive for (5) (not necessarily with the same contraction factor $\lambda$ ). If this is true, then we can conclude that $\Omega$ is an estimate of the RAO of (4) and, consequently, of the RAO of the closed-loop system (1)-(2).

\section{A. Computation of a contractive set for (8)}

The definition of one-step set with respect to (8), given below, plays a key role in this work.

Definition 3: Given $J \in \mathbb{N}$ and $\Omega \subseteq \mathbb{R}^{n}$, the one-step set $Q_{J}(\Omega)$ of $\Omega$ is

$$
Q_{J}(\Omega) \triangleq\left\{x \in \mathbb{R}^{n}: F_{S N S}\left(x, \Delta_{J}, \mathscr{S}\right) \subseteq \Omega\right\} .
$$

Notice that a C-set $\Omega$ is $\lambda$-contractive for (8) if $\Omega \subseteq$ $Q_{J}(\lambda \Omega)$. The following lemma provides a polyhedral characterization of $Q_{J}(\Omega)$ when $\Omega$ is a polyhedron.

Lemma 3: Let $\Omega$ be a polyhedron, where $\Omega=\mathscr{P}(H, h)$ is its $H$-representation [14]. Then

$$
\begin{gathered}
Q_{J}(\Omega)=\bigcap_{\delta \in \Delta_{J}} \bigcap_{S \in \mathscr{S}}\left\{x \in \mathbb{R}^{n}: H\left(A(\delta)+\sum_{i \in S^{c}} B^{(i)}(\delta) K_{(i)}\right) x\right. \\
\left.-\sum_{i \in S}\left|H B^{(i)}(\delta)\right| \leq h\right\}
\end{gathered}
$$

Proof: The proof follows the one in [13, Theorem 1] mutatis mutandis.

The definition below presents the concept of maximal $\lambda$ contractive C-set included in a given C-set $\Omega_{0}$.

Definition 4: Given a C-set $\Omega_{0}$, a $\lambda$-contractive C-set $\Omega \subseteq$ $\Omega_{0}$ is maximal in $\Omega_{0}$ if every $\lambda$-contractive C-set $\Omega^{\prime}$ contained in $\Omega_{0}$ is also contained in $\Omega$.

The following theorem, inspired by [12, Theorem 3.1], introduces the recursion used to find the maximal $\lambda$-contractive $\mathrm{C}$-set for (8) in a given C-set $\Omega_{0}$. Unlike [12, Theorem 3.1], valid only for linear systems, our result applies to the nonlinear case of SNS systems.

Theorem 1: Given $J \in \mathbb{N}$, consider the sequence of sets

$$
\Omega_{i+1}=Q_{J}\left(\lambda \Omega_{i}\right) \cap \Omega_{0}, \forall i \in \mathbb{N},
$$

where $\Omega_{0}$ is a polyhedral C-set and $0<\lambda<1$. The following properties hold:

a) $\Omega_{i}$ is a polyhedral C-set for all $i \in \mathbb{N}$.

b) $\Omega_{i+1} \subseteq \Omega_{i}$ for all $i \in \mathbb{N}$.

c) If a $\lambda$-contractive C-set for (8) $\Omega^{\prime} \subseteq \Omega_{0}$ exists, then

$$
\underline{\Omega}_{\lambda, J} \triangleq \bigcap_{i \in \mathbb{N}} \Omega_{i}
$$

is the maximal $\lambda$-contractive $\mathrm{C}$-set for (8) in $\Omega_{0}$.

Proof:

a) Let us prove it by induction. Assume $\Omega_{i}$ is a polyhedral C-set. Then $\lambda \Omega_{i}$ can be expressed as $\mathscr{P}\left(H_{i}, \mathbf{1}\right)$ for an appropriate choice of $H_{i}$ and it follows from (10) that $Q_{J}\left(\lambda \Omega_{i}\right)$ is a polyhedron containing the origin in its interior. Its intersection with the polytope $\Omega_{0}$ is thus a polytope (which also contains the origin in its interior since $0 \in \Omega_{0}^{\circ}$ ). So $\Omega_{i+1}$ is a polyhedral C-set.

b) This proof also is by induction. If $\Omega_{i+1} \subseteq \Omega_{i}$ (the case $i=0$ is clearly true), then $Q_{J}\left(\lambda \Omega_{i+1}\right) \subseteq Q_{J}\left(\lambda \Omega_{i}\right)$ and it follows that

$$
\Omega_{i+2}=Q_{J}\left(\lambda \Omega_{i+1}\right) \cap \Omega_{0} \subseteq Q_{J}\left(\lambda \Omega_{i}\right) \cap \Omega_{0}=\Omega_{i+1} .
$$

c) Consider any $\lambda$-contractive C-set $\Omega^{\prime \prime} \subseteq \Omega_{0}$ (for instance $\Omega^{\prime \prime}=\Omega^{\prime}$, which exists by hypothesis). It follows by induction that $\Omega^{\prime \prime} \subseteq \underline{\Omega}_{\lambda, J}$. In fact, if $\Omega^{\prime \prime} \subseteq \Omega_{i}$ then

$$
\Omega^{\prime \prime} \subseteq Q_{J}\left(\lambda \Omega^{\prime \prime}\right) \cap \Omega_{0} \subseteq Q_{J}\left(\lambda \Omega_{i}\right) \cap \Omega_{0}=\Omega_{i+1},
$$


i.e. $\Omega^{\prime \prime} \subseteq \bigcap_{i \in \mathbb{N}} \Omega_{i}=\underline{\Omega}_{\lambda, J}$. It also follows that $0 \in \underline{\Omega}_{\lambda, J}^{\circ}$ because $0 \in\left(\Omega^{\prime \prime}\right)^{\circ}$. So $\underline{\Omega}_{\lambda, J}$ is a C-set (compact and convex because it is the intersection of nested C-sets).

We still have to prove $\underline{\Omega}_{\lambda, J}$ is $\lambda$-contractive for (8). Given $x \in \underline{\Omega}_{\lambda, J}$, it follows that $x \in \Omega_{i+1} \subseteq$ $Q_{J}\left(\lambda \Omega_{i}\right), \forall i \in \mathbb{N}$, i.e. $F_{S N S}\left(x, \Delta_{J}, \mathscr{S}\right) \subseteq \lambda \Omega_{i}, \forall i \in \mathbb{N}$. So $F_{S N S}\left(x, \Delta_{J}, \mathscr{S}\right) \subseteq \lambda \bigcap_{i \in \mathbb{N}} \Omega_{i}=\lambda \underline{\Omega}_{\lambda, J}$. Therefore $\underline{\Omega}_{\lambda, J}$ is indeed $\lambda$-contractive.

In general, the set $\underline{\Omega}_{\lambda, J}$ might be not obtainable in a finite number of iterations by an algorithm (and in general it is not polyhedral). That is why we use the following result.

Lemma 4: Assume $\underline{\Omega}_{\lambda, J}$ in (12) is a $\lambda$-contractive $\mathrm{C}$-set for (8). Then for every $\lambda^{*}$ such that $\lambda<\lambda^{*}<1$ there exists $i^{*} \in \mathbb{N}$ such that $\lambda / \lambda^{*} \Omega_{i}$ is $\lambda^{*}$-contractive for (8) for all $i \geq i^{*}$.

Proof: Consider $\lambda^{*} / \lambda>1$. There exists $i^{*} \in \mathbb{N}$ such that $\underline{\Omega}_{\lambda, J} \subseteq \Omega_{i} \subseteq \lambda^{*} / \lambda \underline{\Omega}_{\lambda, J}, \forall i \geq i^{*}[15$, Lemma 1.8.1]. Using these set inclusions (up to scale factors) and the fact that $\underline{\Omega}_{\lambda, J}$ is $\lambda$-contractive for (8) it follows that

$$
\frac{\lambda}{\lambda^{*}} \Omega_{i} \subseteq \underline{\Omega}_{\lambda, J} \subseteq Q_{J}\left(\lambda \underline{\Omega}_{\lambda, J}\right) \subseteq Q_{J}\left(\lambda \Omega_{i}\right)=Q_{J}\left(\lambda^{*}\left(\frac{\lambda}{\lambda^{*}} \Omega_{i}\right)\right)
$$

for all $i \geq i^{*}$, that proves the result.

Lemma 4 ensures that, under the given assumptions, the set $\Omega=\lambda / \lambda^{*} \Omega_{i^{*}}$, obtained by iterating (11) a finite number $i^{*}$ of times, is a $\lambda^{*}$-contractive polyhedral C-set for (8).

\section{B. Testing contractivity for (5)}

The second step of the method consists in verifying if the contractive set found for (8) is also contractive for the dynamics (5), which takes into account all possible values for $\delta_{k} \in \Delta$ and not only the finite set $\Delta_{J}$. The following property plays a key role to verify that.

Lemma 5: Given $d, \tau \in \mathbb{R}$, the following identities hold:

$$
\begin{aligned}
A(d+\tau) & =A(d)+\Phi(\tau) e^{A_{p} d} A_{p} \\
B(d+\tau) & =B(d)+\Phi(\tau)\left(A_{p} \int_{0}^{d} e^{A_{p} s} \mathrm{~d} s B_{p}+B_{p}\right) \\
& =B(d)+\Phi(\tau) e^{A_{p} d} B_{p}
\end{aligned}
$$

where $\Phi(\tau) \triangleq \int_{0}^{\tau} e^{A_{p} s} \mathrm{~d} s$

Proof: See the proof of [6, Proposition 1].

Using the lemma above, it follows that

$$
\begin{aligned}
& F_{S N S}(x, d+\tau, S)=\left[\begin{array}{ll}
A(d+\tau) & B(d+\tau)
\end{array}\right]\left[\begin{array}{c}
x \\
\operatorname{sat}_{S}(K x)
\end{array}\right] \\
& =\left(\left[\begin{array}{ll}
A(d) & B(d)
\end{array}\right]+\Phi(\tau) e^{A_{p} d}\left[\begin{array}{ll}
A_{p} & B_{p}
\end{array}\right]\right)\left[\begin{array}{c}
x \\
\operatorname{sat}_{S}(K x)
\end{array}\right] \\
& =F_{S N S}(x, d, S)+\Phi(\tau) \underbrace{e^{A_{p} d}\left[\begin{array}{ll}
A_{p} & B_{p}
\end{array}\right]}_{\triangleq N(d)}\left[\begin{array}{c}
x \\
\operatorname{sat}_{S}(K x)
\end{array}\right] .
\end{aligned}
$$

Define now the logarithmic norm of $A_{p}$ associated with the 2-norm [16]: $\mu\left(A_{p}\right) \triangleq \lambda_{\max }\left(\frac{A_{p}+A_{p}^{T}}{2}\right)$. Notice in particular that $\mu\left(A_{p}\right)$ can be negative. The following theorem can now be stated.

Theorem 2: Consider $J \in \mathbb{N}$ and a $\lambda^{*}$-contractive polyhedral C-set $\Omega$ for the dynamics (8). If the constant

$$
\bar{c}(\Omega, J) \triangleq c_{1}(J) c_{2} c_{3}(\Omega) c_{4}(\Omega)
$$

where

$$
\begin{gathered}
c_{1}(J) \triangleq\left\{\begin{array}{cc}
\frac{e^{\mu\left(A_{p}\right) \tau_{J}}-1}{\mu\left(A_{p}\right)} & \text { if } \mu\left(A_{p}\right) \neq 0 \\
\tau_{J} & \text { if } \mu\left(A_{p}\right)=0
\end{array}\right. \\
c_{2} \triangleq \max \left(e^{\mu\left(A_{p}\right) \tau_{m}}, e^{\mu\left(A_{p}\right) \tau_{M}}\right) \sqrt{\left\|A_{p}\right\|^{2}+\left\|B_{p}\right\|^{2}} \\
c_{3}(\Omega) \triangleq \max _{x \in \Omega}\left\|\left[\begin{array}{l}
I \\
K
\end{array}\right] x\right\| \\
c_{4}(\Omega) \triangleq \Psi_{\Omega}(\mathscr{B}), \mathscr{B} \triangleq\left\{x \in \mathbb{R}^{n}:\|x\| \leq 1\right\},
\end{gathered}
$$

is such that

$$
v(\Omega, J) \triangleq \lambda^{*}+\bar{c}(\Omega, J)<1,
$$

then $\Omega$ is $v(\Omega, J)$-contractive for the dynamics (5).

Proof: We have to show that $x_{k+1}$ given by (5) satisfies $\Psi_{\Omega}\left(x_{k+1}\right) \leq v(\Omega, J), \forall x_{k} \in \Omega$. Given $x_{k} \in \Omega, \delta_{k} \in \Delta, S_{k} \in \mathscr{S}$, there exist $d_{k} \in \Delta_{J}$ and $\tau_{k} \in\left[0, \tau_{J}\right]$ such that $\delta_{k}=d_{k}+\tau_{k}$. So using (15) it follows that

$$
\begin{aligned}
x_{k+1} & =F_{S N S}\left(x_{k}, d_{k}+\tau_{k}, S_{k}\right) \\
& =F_{S N S}\left(x_{k}, d_{k}, S_{k}\right)+\Phi\left(\tau_{k}\right) N\left(d_{k}\right)\left[\begin{array}{c}
x_{k} \\
\operatorname{sat}_{S_{k}}\left(K x_{k}\right)
\end{array}\right] \\
& \triangleq y_{k+1}+z_{k+1}
\end{aligned}
$$

From the fact that $\Omega$ is $\lambda^{*}$-contractive for (8), $d_{k} \in \Delta_{J}$ and $x_{k} \in \Omega$, it follows that

$$
\Psi_{\Omega}\left(y_{k+1}\right) \leq \Psi_{\Omega}\left(F_{S N S}\left(x_{k}, \Delta_{J}, \mathscr{S}\right)\right) \leq \lambda^{*} .
$$

Considering that $\left\|e^{A_{p} s}\right\| \leq e^{\mu\left(A_{p}\right) s}$ for all $s \geq 0$ (see [16]), and since $\tau_{k} \in\left[0, \tau_{J}\right]$, one obtains:

$$
\left\|\Phi\left(\tau_{k}\right)\right\|=\left\|\int_{0}^{\tau_{k}} e^{A_{p} s} \mathrm{~d} s\right\| \leq \int_{0}^{\tau_{k}}\left\|e^{A_{p} s}\right\| \mathrm{d} s \leq \int_{0}^{\tau_{J}} e^{\mu\left(A_{p}\right) s} \mathrm{~d} s=c_{1}(J) .
$$

Moreover, one has that

$$
\begin{aligned}
\left\|N\left(d_{k}\right)\right\| & \leq\left\|e^{A_{p} d_{k}}\right\|\left\|\left[\begin{array}{ll}
A_{p} & B_{p}
\end{array}\right]\right\| \leq e^{\mu\left(A_{p}\right) d_{k}}\left\|\left[\begin{array}{ll}
A_{p} & B_{p}
\end{array}\right]\right\| \\
& \leq \max _{d \in \Delta_{J}}\left(e^{\mu\left(A_{p}\right) d}\right) \sqrt{\left\|A_{p}\right\|^{2}+\left\|B_{p}\right\|^{2}} \\
& \leq \max \left(e^{\mu\left(A_{p}\right) \tau_{m}}, e^{\mu\left(A_{p}\right) \tau_{M}}\right) \sqrt{\left\|A_{p}\right\|^{2}+\left\|B_{p}\right\|^{2}}=c_{2} .
\end{aligned}
$$

Using the inequalities above we conclude that

$$
\left\|z_{k+1}\right\| \leq\left\|\Phi\left(\tau_{k}\right)\right\|\left\|N\left(d_{k}\right)\right\|\left\|\left[\begin{array}{c}
x_{k} \\
\operatorname{sat}_{S_{k}}\left(K x_{k}\right)
\end{array}\right]\right\| \leq c_{1}(J) c_{2} c_{3}(\Omega),
$$

i.e. $z_{k+1} \in c_{1}(J) c_{2} c_{3}(\Omega) \mathscr{B}$. Then, from (22), (23) and the properties in Lemma 2, we get

$$
\begin{aligned}
\Psi_{\Omega}\left(x_{k+1}\right) & \leq \Psi_{\Omega}\left(y_{k+1}\right)+\Psi_{\Omega}\left(z_{k+1}\right) \leq \lambda^{*}+c_{1}(J) c_{2} c_{3}(\Omega) \Psi_{\Omega}(\mathscr{B}) \\
& =\lambda^{*}+\bar{c}(\Omega, J)=v(\Omega, J) .
\end{aligned}
$$


Remark 1: The constant $c_{3}(\Omega)$ can be obtained, in practice, by taking the maximum over the vertices of the polytope $\Omega$ or by solving a quadratic programming problem with linear constraints. In turn, $c_{4}(\Omega)$ can be computed as

$$
\begin{aligned}
c_{4}(\Omega) & =\Psi_{\Omega}(\mathscr{B})=\min \{\alpha \geq 0: \mathscr{B} \subseteq \alpha \Omega\} \\
& =\min \{\alpha \geq 0: H x \leq \alpha h, \forall x \in \mathscr{B}\}=\max _{i \in \mathbb{N}_{h_{h}}} \frac{\left\|H_{(i)}\right\|}{h_{(i)}}
\end{aligned}
$$

where $\Omega=\mathscr{P}(H, h), H \in \mathbb{R}^{n_{h} \times n}, h \in \mathbb{R}^{n_{h}}$, assuming without loss of generality that $h_{(i)}>0$ for all $i \in \mathbb{N}_{n_{h}}$.

The next theorem makes a connection between the preceding results. It states that, under some conditions, the recursion (11) not only will result in a $\lambda^{*}$-contractive polyhedral $\mathrm{C}$-set $\Omega=\lambda / \lambda^{*} \Omega_{i^{*}}$ for (8) but also that this set $\Omega$ will indeed satisfy the hypothesis (21) of Theorem 2 .

Theorem 3: Assume that there exists a $\gamma$-contractive C-set $\Omega^{\prime} \subseteq \Omega_{0} \subset \mathbb{R}^{n}$ for (5), where $\Omega_{0}$ is a polyhedral C-set and $0<\gamma<1$. Then, given $\lambda, \lambda^{*}$ with $\gamma \leq \lambda<\lambda^{*}<1$, there exists $\bar{J}=\bar{J}\left(\lambda, \lambda^{*}\right)$ satisfying the following property: for all $J \geq \bar{J}$ there is $i^{*} \in \mathbb{N}$ such that the set $\Omega=\lambda / \lambda^{*} \Omega_{i^{*}}$ obtained by the recursion (11) is $\lambda^{*}$-contractive for (8). Moreover, for such $i^{*}$ the constraint (21) will be satisfied for $\Omega$.

Proof: Since $\Omega^{\prime}$ is $\gamma$-contractive for (5) and $\lambda \geq \gamma$ it is also $\lambda$-contractive for (8) for every $J \in \mathbb{N}$. So from item c) of Theorem 1 it follows that $\Omega^{\prime} \subseteq \underline{\Omega}_{\lambda, J}$ and that $\underline{\Omega}_{\lambda, J}$ is a $\lambda$-contractive $\mathrm{C}$-set for (8). Applying now Lemma 4 we conclude that there exists $i^{*} \in \mathbb{N}$ such that $\Omega=\lambda / \lambda^{*} \Omega_{i^{*}}$ is $\lambda^{*}$-contractive for (8), i.e. $\Omega \subseteq Q_{J}\left(\lambda^{*} \Omega\right)$.

We still have to show that $v(\Omega, J)=\lambda^{*}+\bar{c}(\Omega, J)<1$ for all $J \geq \bar{J}$, where $\bar{J}=\bar{J}\left(\lambda, \lambda^{*}\right)$ must be appropriately chosen. Recalling that $\Omega=\lambda / \lambda^{*} \Omega_{i^{*}} \subset \Omega_{i^{*}} \subseteq \Omega_{0}$, we have that:

$$
\bar{c}_{3} \triangleq \max _{x \in \Omega_{0}}\left\|\left[\begin{array}{l}
I \\
K
\end{array}\right] x\right\| \geq \max _{x \in \Omega}\left\|\left[\begin{array}{l}
I \\
K
\end{array}\right] x\right\|=c_{3}(\Omega) .
$$

Moreover, as $\lambda / \lambda^{*} \Omega^{\prime} \subseteq \lambda / \lambda^{*} \underline{\Omega}_{\lambda, J} \subseteq \lambda / \lambda^{*} \Omega_{i^{*}}=\Omega$ :

$$
\bar{c}_{4} \triangleq \Psi_{\lambda / \lambda^{*} \Omega^{\prime}}(\mathscr{B}) \geq \Psi_{\Omega}(\mathscr{B})=c_{4}(\Omega) .
$$

Since $c_{1}(J) \stackrel{J \rightarrow \infty}{\rightarrow} 0$, there exists $\bar{J}=\bar{J}\left(\lambda, \lambda^{*}\right)$ such that $c_{1}(J) c_{2} \bar{c}_{3} \bar{c}_{4}<\left(1-\lambda^{*}\right)$ for all $J \geq \bar{J}$. Thus for every $J \geq \bar{J}$

$$
\begin{gathered}
v(\Omega, J)=\lambda^{*}+\bar{c}(\Omega, J)=\lambda^{*}+c_{1}(J) c_{2} c_{3}(\Omega) c_{4}(\Omega) \\
\underbrace{\leq}_{(24),(25)} \lambda^{*}+c_{1}(J) c_{2} \bar{c}_{3} \bar{c}_{4}<1
\end{gathered}
$$

which concludes the proof.

Remark 2: As a byproduct of the proof, notice that the existence of a $\gamma$-contractive C-set $\Omega^{\prime} \subseteq \Omega_{0} \subset \mathbb{R}^{n}$ for (5) not only guarantees that the proposed recursion (11) will result in a $v(\Omega, J)$-contractive polytope $\Omega$ for (5), with appropriate choices of $\lambda, \lambda^{*}$ and $J$, but also ensures that the obtained estimate of the RAO $\Omega$ will include the estimate $\Omega^{\prime}$ up to a scaling factor $\lambda / \lambda^{*}$, i.e. $\lambda / \lambda^{*} \Omega^{\prime} \subseteq \Omega$.

\section{Numerical ALGORITHM}

The Algorithm 1 below is proposed to obtain a $v(\Omega, J)$ contractive polyhedral C-set $\Omega$ for (5), and can be therefore used as an estimate of the RAO of (1)-(2).

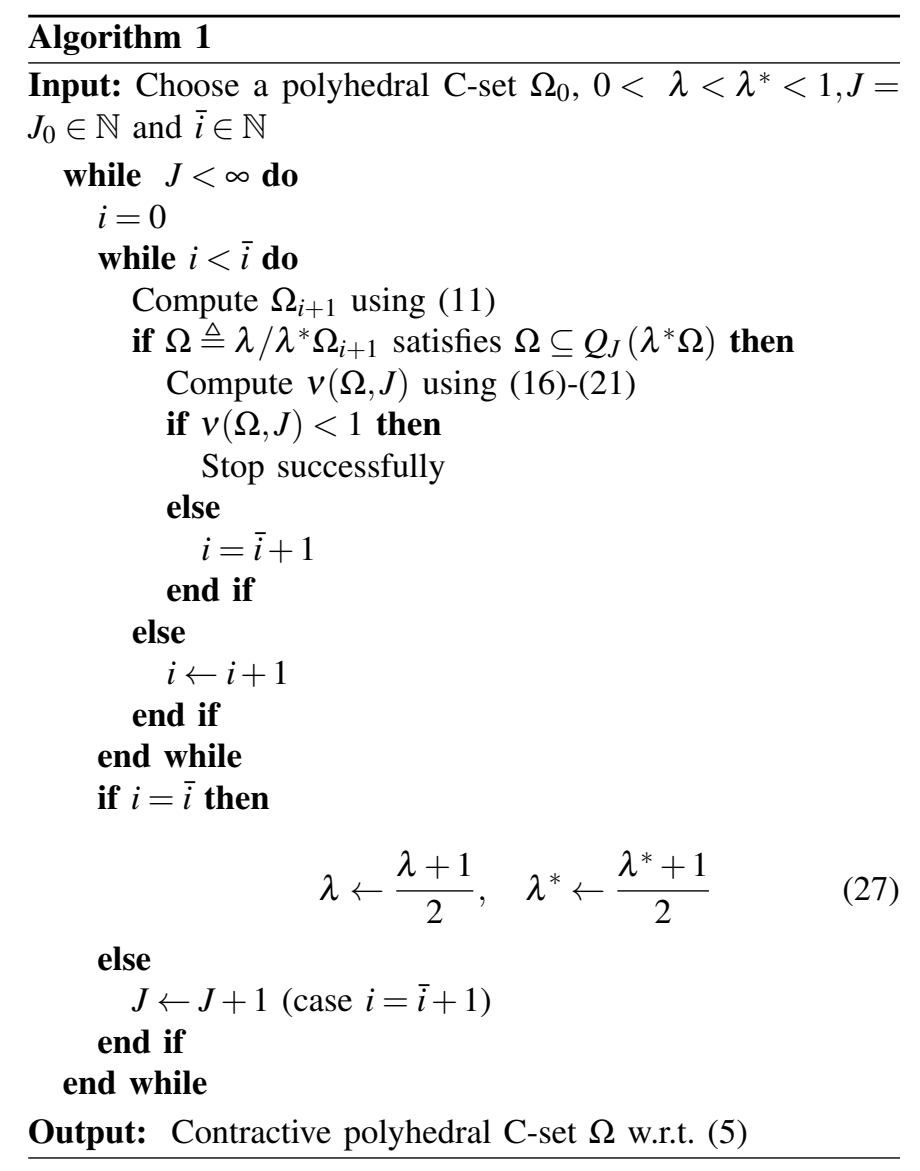

Notice that (11) is recursively applied until a $\lambda^{*}$-contractive polytope is found for (8) or until the maximum number of iterations $\bar{i}$ is reached, with $\bar{i}$, empirically chosen, assumed to be a very large constant (such that $\bar{i}>i^{*}$ will most likely be true where $i^{*}$ is defined in Lemma 4). If $\bar{i}$ is reached, the algorithm increases the value of $\lambda$ with the aim of making $\lambda \geq \gamma$ according to the statement of Theorem 3, where $\gamma$ is not known a priori. The update rule (27) is just a possible choice and can be modified by the users of the method.

On the other hand, if a $\lambda^{*}$-contractive polytope is found for (8) but constraint (21) is violated, the algorithm increases the value of $J$. This choice is also inspired by the assumptions of Theorem 3, which guarantees that $v(\Omega, J)<1$ for $J$ sufficiently large if the other hypothesis hold.

\section{NUMERICAL EXAMPLES}

\section{A. Example /}

Consider the system taken from [11], where:

$$
A_{p}=\left[\begin{array}{ll}
0 & 1 \\
1 & 0
\end{array}\right], B_{p}=\left[\begin{array}{c}
0 \\
-5
\end{array}\right], K=\left[\begin{array}{ll}
2.6 & 1.4
\end{array}\right], \Delta=[0.05,0.1] \text {. }
$$

Choosing $\lambda=0.98$ and $\lambda^{*}=0.99$ and considering as initial set $\Omega_{0}$ a square with side of size 20 centered at the origin, the algorithm above gives, for $J=140$, the polytope $\Omega_{35}$ displayed in Figure 1, which is an estimate of the RAO of (1)-(2). In this case, the contractivity factor of $\Omega_{35}$ is given by $v\left(\Omega_{35}, 140\right) \cong 0.999<1$. For comparison purposes, the figure also shows the piecewise quadratic estimate obtained with 
the conditions proposed in [11] and the ellipsoidal estimates obtained through the methods presented in [10] and [17]. Notice that the approach presented here results in an estimate of the RAO that includes the other ones.

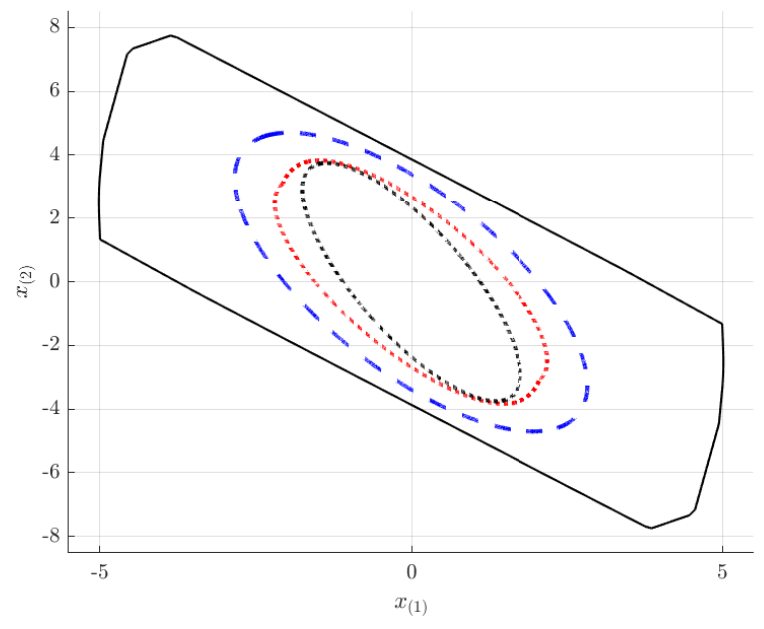

Fig. 1. Estimates of the RAO of (1)-(2) given by the proposed approach (black-continuous) and by the methods proposed in [10] (black-dotted), [11] (red-dotted) and [17] (blue-dashed).

\section{B. Example II}

Consider the three-dimensional system (1)-(2) with:

$$
\begin{aligned}
A_{p} & =\left[\begin{array}{ccc}
0.75 & 0.35 & 1.75 \\
0.7 & 0 & 0.7 \\
0.75 & -1.1 & 1.75
\end{array}\right], & B_{p}=\left[\begin{array}{c}
0.7 \\
0 \\
0.7
\end{array}\right], \\
K & =\left[\begin{array}{lll}
-24.82 & -22.85 & 11.13
\end{array}\right], & \Delta=[0.1,0.2] .
\end{aligned}
$$

Choosing $\lambda=0.8$ and $\lambda^{*}=0.95$ and considering as initial

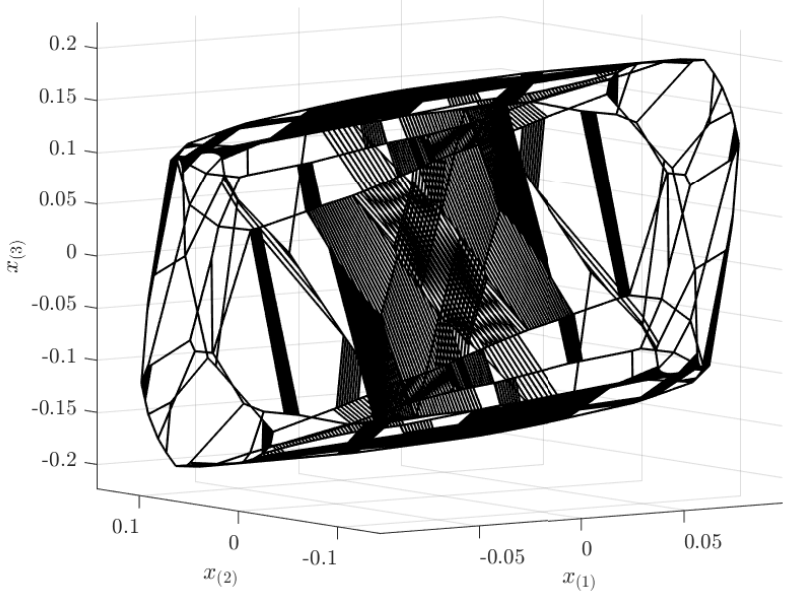

Fig. 2. Estimate of the RAO of (1)-(2) given by the proposed approach.

set $\Omega_{0}$ a square with side of size 20 centered at the origin, the algorithm leads, for $J=100$, to the polytope $\Omega_{12}$ displayed in Figure 2 , where $v\left(\Omega_{12}, 100\right) \cong 0.99<1$.

\section{CONCLUSIONS}

A method for the computation of polyhedral estimates of the RAO of aperiodic sampled-data systems subject to control input saturation was presented. As shown in the first example, the obtained polytope provided a less conservative estimate of the RAO than other methods in the literature. The main drawback of our approach is its numerical complexity, as usual for methods based on polyhedrons [14]. On the other hand, efficient numerical tools for dealing with this class of sets exist, as the MPT toolbox [18], for instance.

\section{REFERENCES}

[1] J. P. Hespanha, P. Naghshtabrizi, and Y. Xu, "A survey of recent results in networked control systems," Proceedings of the IEEE, vol. 95, no. 1, pp. 138-162, 2007.

[2] L. Hetel, C. Fiter, H. Omran, A. Seuret, E. Fridman, J.-P. Richard, and S. I. Niculescu, "Recent developments on the stability of systems with aperiodic sampling: An overview," Automatica, vol. 76, pp. 309 - 335, 2017.

[3] L. Hetel, J. Daafouz, J. Richard, and M. Jungers, "Delay-dependent sampled-data control based on delay estimates," Systems \& Control Letters, vol. 60, no. 2, pp. 146 - 150, 2011.

[4] M. Cloosterman, N. V. D. Wouw, W. Heemels, and H. Nijmeijer, "Stability of networked control systems with uncertain time-varying delays," IEEE Trans. on Automatic Control, vol. 54, no. 7, pp. 1575$1580,2009$.

[5] M. Cloosterman, L. Hetel, N. V. D. Wouw, W. Heemels, J. Daafouz, and H. Nijmeijer, "Controller synthesis for networked control systems," Automatica, vol. 46, no. 10, pp. 1584-1594, 2010.

[6] H. Fujioka, "A discrete-time approach to stability analysis of systems with aperiodic sample-and-hold devices," IEEE Transactions on Automatic Control, vol. 54, no. 10, pp. 2440-2445, 2009.

[7] H. Fujioka and Y. Oishi, "A switched Lyapunov function approach to stability analysis of non-uniformly sampled-data systems with robust LMI techniques," in 8th Asian Control Conference (ASCC), pp. 14871491,2011

[8] M. Fiacchini and I.-C. Morărescu, "Constructive necessary and sufficient condition for the stability of quasi-periodic linear impulsive systems," IEEE Transactions on Automatic Control, vol. 61, no. 9, pp. 2512-2517, 2016.

[9] S. Tarbouriech, G. Garcia, J. M. Gomes da Silva Jr., and I. Queinnec, Stability and Stabilization of Linear Systems with Saturating Actuators. Springer, 2011.

[10] A. Seuret and J. M. Gomes da Silva Jr., "Taking into account period variations and actuator saturation in sampled-data systems," Systems \& Control Letters, vol. 61, pp. 1286-1293, 2012.

[11] M. Fiacchini and J. M. Gomes da Silva Jr., "Stability of sampled-data control systems under aperiodic sampling and input saturation," in 57th IEEE Conference on Decision and Control (CDC), pp. 6644-6649, 2018.

[12] F. Blanchini, "Ultimate boundedness control for uncertain discrete-time systems via set-induced lyapunov functions," IEEE Transactions on Automatic Control, vol. 39, no. 2, pp. 428-433, 1994.

[13] T. Alamo, A. Cepeda, D. Limon, and E. Camacho, "A new concept of invariance for saturated systems," Automatica, vol. 42, no. 9, pp. 1515 - 1521, 2006.

[14] F. Blanchini and S. Miani, Set-Theoretic Methods in Control. Birkhäuser, 2015.

[15] R. Schneider, Convex Bodies: The Brunn-Minkowski Theory. Encyclopedia of Mathematics and its Applications, Cambridge University Press, 2 ed., 2013

[16] C. Van Loan, "The sensitivity of the matrix exponential," SIAM Journal on Numerical Analysis, vol. 14, no. 6, pp. 971-981, 1977.

[17] D. D. Huff, M. Fiacchini, and J. M. Gomes da Silva Jr, "Stability and stabilization of sampled-data systems subject to control input saturation: a set invariant approach," To appear on IEEE Transactions on Automatic Control, DOI:10.1109/TAC.2021.3064988, 2021.

[18] M. Herceg, M. Kvasnica, C. Jones, and M. Morari, "Multi-Parametric Toolbox 3.0," in Proc. of the European Control Conference, pp. 502510, 2013. 